\title{
Epidemiological study of hemotropic mycoplasmas (hemoplasmas) in cats from central Spain
}

David Díaz-Regañón ${ }^{1}$, Alejandra Villaescusa', Tania Ayllón², Fernando Rodríguez-Franco ${ }^{1}$, Mercedes García-Sancho', Beatriz Agulla ${ }^{1}$ and Ángel Sainz ${ }^{1 *}$

\begin{abstract}
Background: Hemotropic mycoplasmas (hemoplasmas) have been found infecting cats worldwide. However, studies about feline hemoplasma infections in Spain are scarce. Therefore, the purpose of the research was to evaluate the prevalence of feline hemotropic mycoplasmas and to characterize risk factors and clinical findings associated with these infections in a cat population from the Madrid area, Spain.
\end{abstract}

Methods: Polymerase chain reaction (PCR) was employed to detect Mycoplasma haemofelis (Mhf), "Candidatus Mycoplasma haemominutum" (CMhm) and "Candidatus Mycoplasma turicensis" (CMt) in blood samples from 456 client-owned and 138 stray cats from Madrid. In order to assess associations between these hemoplasma infections and epidemiological parameters, data regarding signalment, environment, prophylaxis measures, retrovirus status, clinical signs and laboratory findings were compiled, whenever possible.

Results: DNA of feline hemoplasmas was detected from the blood of 63 out of 594 cats (10.6\%), with a prevalence of 3.7\% (22/594) for Mhf, 8.1\% (48/594) for CMhm and 0.5\% (3/594) for CMt. Stray cats had statistically higher prevalences of feline hemoplasmas (15.9\%) and, specifically, of Mhf (8.7\%) than client-owned cats (9 and 2.2\%, respectively). A total of seven cats (1.17\%) were co-infected with "Candidatus $\mathrm{M}$. haemominutum" and $\mathrm{M}$. haemofelis, two (0.33\%) with "Candidatus M. haemominutum" and "Candidatus M. turicensis" and another one (0. 17\%) with M. haemofelis and Candidatus "M. turicensis". Male gender, collection of blood during warm months and FeLV/FIV positivity status were associated with hemotropic mycoplasma infection in cats from Madrid. Additionally, within the group of client-owned cats, hemoplasma infection was associated with adult age, outdoor access, and the existence of low haematocrit, erythrocyte count and haemoglobin concentration values.

Conclusions: To our knowledge, this is the first epidemiological survey of feline hemoplasmas performed in central Spain (Madrid). Our study confirms that "Ca. Mycoplasma haemominutum", Mycoplasma haemofelis and "Ca. Mycoplasma turicensis" are infecting client-owned and stray cats in this region of Spain, "Ca. Mycoplasma haemominutum" being the most prevalent species. More studies are necessary to help understand the role of the natural infection by these species of hemoplasma in cats.

Keywords: Hemotropic mycoplasmas, Hemoplasmas, Cats, Central Spain, Polymerase chain reaction

\footnotetext{
* Correspondence: angelehr@vet.ucm.es

${ }^{1}$ Department of Animal Medicine and Surgery, College of Veterinary

Medicine, Complutense University of Madrid, Avda. Puerta de Hierro s/n,

28040 Madrid, Spain

Full list of author information is available at the end of the article
} 


\section{Background}

Hemotropic mycoplasmas (the so-called hemoplasmas) are small wall-less bacteria that attach to the erythrocytes [1], causing anaemia in different mammalian species, including cats. Three hemoplasma species have been typically detected in cats: Mycoplasma haemofelis (Mhf) [2], "Candidatus Mycoplasma haemominutum" (CMhm) [3] and "Candidatus Mycoplasma turicensis" (CMt) [4]. Another species, "Candidatus Mycoplasma haematoparvumlike", has also been reported in cats [5-7]. Although these bacteria are distributed worldwide, the prevalence varies geographically [7-11].

It is still unknown how feline hemoplasmas are transmitted. Vector transmission through fleas [12-16] or ticks $[17,18]$ has been suggested, but direct transmission through aggressive interactions or blood transfusion have also been hypothesized as potential sources of infection.

Clinical presentation varies from absence of clinical signs to the existence of acute haemolytic anaemia, showing the affected cats pallor, depression, lethargy, weight loss, anorexia, dehydration and intermittent pyrexia or even sudden death [1]. In this sense, Mhf seems to be the most pathogenic of the three main feline hemoplasmas. The clinical presentation can vary depending not only on the pathogenicity of the haemoplasma species, but also on host factors, such as the presence of concurrent disease. Younger cats are more susceptible to clinical haemoplasmosis [19]. Other factors such as infecting organisms' dose or route of infection may also impact on outcome [1].

There are just a few studies reporting hemoplasma infection in cats in Spain $[9,20,21]$ and, to our knowledge, no epidemiological studies on these bacteria have been performed in the central region of the country. The objective of this study was to determine the prevalence of feline hemoplasmas (Mhf, CMhm and CMt) in cats from Madrid, central Spain, and to characterize risk factors and clinical signs associated with these feline infections in the area.

\section{Methods}

\section{Recruitment and data collection}

During a three-year period, blood samples were collected from 456 client-owned cats admitted to the Veterinary Medicine Teaching Hospital (VMTH) of the Complutense University of Madrid and 138 stray cats attended to be neutered/spayed in different clinics or animal protection societies located in the Community of Madrid. Client-owned cats from which blood samples were collected were not subjected to any other inclusion or exclusion criteria.

Data obtained at the time of enrolment of the clientowned cats in the study included signalment, environmental and lifestyle characteristics, such as living in urban/periurban/rural areas, travel history, and outdoor access; contact with other animals, exposure to arthropods and administration of ectoparasiticides; and clinical data, including previous tetracyclines treatment (in the last 60 days) or blood transfusions. Data collected from cats from animal protection societies were scarce, and included gender, living area and FeLV/FIV status. In addition, the date on which blood sample was drawn was recorded for all the cats included in this study.

Feline leukemia virus (FeLV) and feline immunodeficiency virus (FIV) status were tested using a commercial kit (IDEXX Laboratories, Inc., Westbrook, Maine, USA) and clinical signs and laboratory findings (erythrocyte, leukocyte and platelets count, haemoglobin, haematocrit) were also recorded. Feline infectious peritonitis (FIP) status was also checked using a commercial inhouse antibody test (ImmunoComb FCoV Antibody Test Kit, Biogal, Kibbutz Galed, Israel) to detect seropositive feline coronavirus (FCoV).

\section{Nucleic acid extraction, quality control and PCR amplification}

An UltraCleanTM DNA Blood Spin Kit (Mo Bio Laboratories, Carlsbad, USA) was employed for the DNA extraction from whole blood. Before the PCR amplification, DNA was evaluated for quality at 260/280 $\mathrm{nm}$ and quantified at 260/230 nm using a NanoDrop ${ }^{\mathrm{TM}}$ spectrophotometer (Thermo Scientific, Waltham, USA).

A total of 594 samples of genomic DNA were processed, using the PCR-protocol [8] based on the amplification of a partial sequence of the $16 S$ rRNA gene of feline hemotrophic mycoplasmas. As previously described by Kamrani et al. [12], amplified products of 193 bp were identified as CMhm, and products of $170 \mathrm{bp}$ as Mhf or CMt. Thus, a second PCR protocol [11] was carried out using specific CMt primers in all the previous PCR-positive samples.

The sequences of the primers employed, and PCR protocols are shown in Table 1 . A total of $5 \mu \mathrm{l}$ of genomic DNA was added to $12.5 \mu \mathrm{l}$ Premix Ex Taq ${ }^{\mathrm{TM}}$ (Conda, Madrid, Spain), $7.5 \mu \mathrm{l}$ of deionized sterile water and 0.25 $\mu \mathrm{l}(100 \mu \mathrm{M})$ of the primers in a $25.5 \mu \mathrm{l}$ final volume of the reaction mixture. The reactions were carried out in an automatic DNA thermal cycler MasterCycler ep Gradient (Eppendorf, Hamburg, Germany), including every time negative and positive controls. The PCR amplification products were visualized by ethidium bromide fluorescence after electrophoresis in a $3 \%$ agarose gel at $115 \mathrm{~V}$ for $30 \mathrm{~min}$ for the first PCR and 1.5\% agarose gel at $90 \mathrm{~V}$ for the second one.

Purification of amplified DNA fragments obtained for the first PCR was obtained using the Speed Clean-Up kit (Biotools, Madrid, Spain) and subsequently sequenced in 
Table 1 Primers and protocols used for the amplification of feline hemoplasmas and housekeeping GAPDH gene control

\begin{tabular}{|c|c|c|c|}
\hline Target gene & $\begin{array}{l}\text { Product size } \\
\text { (bp) }\end{array}$ & PCR primers $\left(5^{\prime}-3^{\prime}\right)$ & PCR conditions \\
\hline \multirow[t]{2}{*}{ 16S rRNA hemoplasmas [8] } & \multirow[t]{2}{*}{$170 / 193$} & F: ACGAAAGTCTGATGGAGCAATA & \multirow{2}{*}{$\begin{array}{l}94{ }^{\circ} \mathrm{C}, 1 \mathrm{~min} ; 45 \text { cycles }\left[94^{\circ} \mathrm{C}, 1 \mathrm{~min} ; 65^{\circ} \mathrm{C}, 1 \mathrm{~min} ; 72{ }^{\circ} \mathrm{C}, 30 \mathrm{~s}\right] \\
72{ }^{\circ} \mathrm{C}, 10 \mathrm{~min}\end{array}$} \\
\hline & & R: ACGCCCAATAAATCCGRATAAT & \\
\hline \multirow[t]{2}{*}{ CMt [11] } & \multirow[t]{2}{*}{138} & F: AGAGGCGAAGGCGAAAACT & \multirow{2}{*}{$\begin{array}{l}95^{\circ} \mathrm{C}, 2 \mathrm{~min} ; 30 \text { cycles }\left[95^{\circ} \mathrm{C}, 10 \mathrm{~s} ; 58^{\circ} \mathrm{C}, 30 \mathrm{~s} ; 72^{\circ} \mathrm{C}, 30 \mathrm{~s}\right] ; \\
72^{\circ} \mathrm{C} 5 \mathrm{~min}\end{array}$} \\
\hline & & R: CTACAACGCCGAAACACAAA & \\
\hline \multirow[t]{2}{*}{ GAPDH-Housekeeping [22] } & \multirow[t]{2}{*}{282} & F: CCTTCATTGACCTCAACTACAT & \multirow{2}{*}{$\begin{array}{l}95^{\circ} \mathrm{C}, 1 \mathrm{~min} ; 45 \text { cycles }\left[94^{\circ} \mathrm{C}, 4 \mathrm{~s} ; 57^{\circ} \mathrm{C}, 4 \mathrm{~s} ; 72{ }^{\circ} \mathrm{C}, 3 \mathrm{~s}\right] ; \\
72{ }^{\circ} \mathrm{C}, 1 \mathrm{~min}\end{array}$} \\
\hline & & R: CCAAAGTTGTCATGGATGACC & \\
\hline
\end{tabular}

Abbreviations: CMt "Candidatus Mycoplasma turicensis", GAPDH gliceraldehide-3-phosphate dehydrogenase

an automated 3730 DNA Analyzer using a Big Dye Terminator 3.1 (Applied Biosystems, Foster City, USA) in Secugen S.L. (Madrid, Spain). Data obtained were compared with reference sequences deposited in GenBank, using the Basic Local Alignment Search Tool (BLAST).

Negative DNA samples were submitted to internal control PCR to evaluate both the presence of the amplifiable DNA and the absence of inhibitor factors [22].

\section{Statistical analysis}

Statistical associations between results obtained by PCR and epidemiological, and hematological data were analyzed in the case of client-owned cats using the Chisquare test or Fisher's exact test, where appropriate, and odds ratio (OR) with a $95 \%$ CI. It was possible to perform these statistical analyses for the whole feline sample (stray and client-owned cats) for data regarding gender, living area, months of sample collection and FeLV/FIV status. Other epidemiological information (related to signalment, environment, prophylaxis measures, clinical signs and laboratory findings) was only available for client-owned cats. Thus, statistical associations between PCR results and these data were assessed only for this group of client-owned cats. The significance level was established at $P<0.05$. In order to exclude any possible confounding factors, logistic regression analysis with backward elimination was complementary performed with those variables that showed a statistical association with any hemoplasma infection. Analysis of the data was carried out with the support of "Departamento de Ayuda a la Investigación, Ârea de Informática y Comunicaciones", Complutense University of Madrid, employing the statistical software SAS, 9.4 (SAS Institute, Cary, NC, USA).

\section{Results}

Out of the 594 cats included in this study, 138 (23.2\%) were stray cats and $456(76.8 \%)$ were client-owned cats. It was not possible to obtain epidemiological and/or clinical data from every cat involved in the study, especially in stray animals. Table 2 shows the information available from the whole sample of cats included in this study (stray and client-owned cats). Data gathered from the population of the client-owned cats are shown in Tables 3 and 4. In addition, these tables show the prevalences for the different hemoplasma species and the results for the assessment of associations between positivity to the hemoplasma PCR and the rest of data.

The overall prevalence of hemoplasma infection in cats from Madrid was 10.6\% (63/594). The prevalences of CMhm, Mhf and CMt were 8.1\% (48/594), $3.7 \%(22 / 594)$ and $0.5 \%(3 / 594)$, respectively. A total of seven cats $(1.17 \%)$ were co-infected with $\mathrm{CMhm}$ and Mhf, two $(0.33 \%)$ with CMhm and CMt and another one $(0.17 \%)$ with Mhf and CMt. No single infection with CMt was detected.

Attending to the different lifestyle groups, prevalence of hemoplasma infection in stray cats was $15.9 \%$ (22/ 138) and prevalence in client-owned cats was $9 \%(41 /$ 456), the lifestyle (stray) of the cat being statistically associated with hemoplasma infection $\left(\chi^{2}=5.40, d f=1, P\right.$ $=0.020)$. The same was observed when attending to Mhf infection $\left(\chi^{2}=12.56, d f=1, P=0.0004\right)$, with $8.7 \%$ (12/ 138) positive to Mhf in stray cats, and $2.2 \%(10 / 456)$ in client-owned cats, but not in the case of CMhm or CMt. Data related to prevalence in different lifestyle groups are shown in Table 2.

Considering any of the hemoplasma infections assessed in stray and client-owned cats of this study, the season of the sample collection was significantly associated with positivity by PCR. Specifically, 63.5\% (40/63) of the positive samples for any of the hemoplasmas were collected during spring and summer $\left(\chi^{2}=6.21, d f=1, P\right.$ $=0.013)$. When considering this association in the different species of the study separately, the same was observed for CMhm $\left(\chi^{2}=4.01, d f=1, P=0.045\right)$, with $62.5 \%(30 / 48)$ samples drawn in spring or summer, while Mhf infection was detected more frequently specifically in the samples collected during summer months in comparison with the other seasons together $(P=0.032$, OR $=0.35$; $95 \%$ CI: 0.13-0.89).

Within other epidemiological data available from stray and client-owned cats of the study, significant associations were detected between male gender and CMhm 
Table 2 Comparison of prevalences of hemoplasma infection and epidemiological data in stray and client-owned cats

\begin{tabular}{|c|c|c|c|c|c|c|c|c|}
\hline \multirow[t]{2}{*}{ Variable } & \multirow{2}{*}{$\begin{array}{l}\text { Total no. } \\
\text { of cats } \\
(\%)\end{array}$} & \multicolumn{7}{|c|}{ Number of positive cats (\%) } \\
\hline & & Any hemoplasma & Mhf & CMhm & $\mathrm{CMt}$ & $\mathrm{Mhf}+\mathrm{CMhm}$ & $\mathrm{CMt}+\mathrm{CMhm}$ & $\mathrm{CMt}+\mathrm{Mht}$ \\
\hline Lifestyle & 594 & $63(10.6)$ & $22(3.7)$ & $48(8.1)$ & $3(0.5)$ & $7(1.2)$ & $2(0.3)$ & $1(0.2)$ \\
\hline Client-owned & $456(76.8)$ & $41(9.0)$ & $10(2.2)$ & $36(7.9)$ & $2(0.4)$ & $5(1.1)$ & $1(0.2)$ & $1(0.2)$ \\
\hline Stray & $138(23.2)$ & $22(15.9)^{*}$ & $12(8.7)^{*}$ & $12(8.7)$ & $1(0.7)$ & $2(1.4)$ & $1(0.7)$ & 0 \\
\hline Months of sample collection & 594 & & & & & & & \\
\hline Warm months & $289(48.6)$ & $40(13.8)^{*}$ & $15(5.2)$ & $30(10.4)^{*}$ & $2(0.7)$ & $5(1.7)$ & $2(0.7)$ & 0 \\
\hline Cold months & $305(51.4)$ & $23(7.5)$ & $7(2.3)$ & $18(5.9)$ & $1(0.3)$ & $2(0.6)$ & 0 & $1(0.3)$ \\
\hline Gender & 540 & & & & & & & \\
\hline Male & $260(51.8)$ & $42(16.1)^{*}$ & $15(5.8)^{*}$ & $32(12.3)^{*}$ & $2(0.8)$ & $5(1.9)$ & $1(0.4)$ & $1(0.4)$ \\
\hline Female & $280(48.2)$ & $12(4.3)$ & $4(1.4)$ & $8(2.9)$ & 0 & 0 & 0 & 0 \\
\hline Living area & 394 & & & & & & & \\
\hline Urban & $223(56.6)$ & $11(4.9)$ & $3(1.3)$ & $10(4.5)$ & 0 & $2(0.9)$ & 0 & 0 \\
\hline Periurban & $99(25.1)$ & $10(10.1)$ & $2(2.0)$ & $9(9.1)$ & $1(1.0)$ & $1(1.0)$ & $1(1.0)$ & 0 \\
\hline Rural & $72(18.3)$ & $8(11.1)$ & $2(2.8)$ & $6(8.3)$ & $1(1.4)$ & 0 & 0 & $1(1.4)$ \\
\hline FeLV & 445 & & & & & & & \\
\hline Yes & $32(7.2)$ & $8(25.0)^{*}$ & $3(9.4)$ & $6(18.7)^{*}$ & 0 & $1(3.1)$ & 0 & 0 \\
\hline No & $413(92.8)$ & $38(9.2)$ & $16(3.9)$ & $27(6.5)$ & $2(0.5)$ & $5(1.2)$ & $1(0.2)$ & $1(0.2)$ \\
\hline FIV & 447 & & & & & & & \\
\hline Yes & $23(5.2)$ & $10(43.5)^{*}$ & $5(21.7) *$ & $6(26.1)^{*}$ & $1(4.3)$ & $1(4.3)$ & 0 & $1(4.3)$ \\
\hline No & $424(94.8)$ & $37(8.3)$ & $14(3.3)$ & $28(6.6)$ & 0 & $5(1.2)$ & 0 & 0 \\
\hline
\end{tabular}

Abbreviations: CMhm "Candidatus Mycoplasma haemominutum", Mhf Mycoplasma haemofelis, CMt "Candidatus Mycoplasma turicensis"

*Statistically significant differences $(P<0.05)$

$\left(\chi^{2}=17.55, d f=1, P<0.0001\right), \operatorname{Mhf}\left(\chi^{2}=7.48, d f=1, P\right.$ $=0.006)$ and overall hemoplasma infection $\left(\chi^{2}=21.10\right.$, $d f=1, P<0.0001)$.

When analyzing epidemiological information that was available from client-owned cats only, some additional statistically significant associations between these data and hemoplasma prevalence were detected. Clientowned cats older than one year of age showed a significantly greater risk for hemoplasma infection $\left(\chi^{2}=4.41\right.$, $d f=1, P=0.036)$. This finding was not found when considering CMhm $\left(\chi^{2}=3.40, d f=1, P=0.065\right)$, or $\operatorname{Mhf}(P$ $=0.665, \mathrm{OR}=0.75 ; 95 \% \mathrm{CI}: 0.15-3.79)$ infection alone.

Having an outdoor access was identified as an additional risk factor for any hemoplasma species in clientowned cats $\left(\chi^{2}=5.51, d f=1, P=0.019\right)$. None of the other epidemiological data evaluated were associated with hemoplasma infection.

Retroviral status was confirmed as a risk factor in stray and client-owned cats. Cats positive for FeLV were more likely to be infected with any hemoplasma $(P=0.011$, $\mathrm{OR}=8.04 ; 95 \% \mathrm{CI}: 3.3-19.6)$ and with CMhm $(P=$ $0.023, \mathrm{OR}=3.3 ; 95 \% \mathrm{CI}: 1.25-8.7)$. In a similar way, FIV-infected cats exhibited higher prevalences of CMhm $(P=0.005$, OR $=4.99 ; 95 \% \mathrm{CI}: 1.82-13.65)$, Mhf $(P=$ $0.002, \mathrm{OR}=8.13 ; 95 \% \mathrm{CI}: 2.64-25.06)$ and overall hemoplasma infection $(P<0.0001$, OR $=8.04$; $95 \% \mathrm{CI}$ : 3.3-19.61).

No statistically significant differences in hemoplasmas' prevalences were detected between symptomatic and asymptomatic client-owned cats (Table 4). However, when specific clinical signs were evaluated, muscle-skeletal $(15.8 \%, 6 / 38)$ and renal $(11.2 \%, 13 / 116)$ signs were associated with $\mathrm{CMhm}$ infection $(P=0.035, \mathrm{OR}=2.97 ; 95 \% \mathrm{CI}$ : $1.13-7.82$, and $\chi^{2}=4.82, d f=1, P=0.028$, respectively).

Hemoplasma-infected cats showed some remarkable findings in the hematological analysis. Low red blood cell $(\mathrm{RBC})$ count was associated with infection by any hemoplasma $(P=0.009$, OR $=0.22$; 95\% CI: $0.07-0.62)$, and specifically with $\mathrm{Mhf}(P=0.031, \mathrm{OR}=0.09 ; 95 \% \mathrm{CI}$ : $0.01-0.56)$ and CMhm $(P=0.025, \mathrm{OR}=0.26$; $95 \% \mathrm{CI}$ : 0.08-0.78) infection. Low haemoglobin (HGB) concentration was significantly associated with any hemoplasma and CMhm infection $\left(\chi^{2}=9.21, d f=1, P=0.002\right.$ and $\chi^{2}$ $=8.86 d f=1, P=0.003$, respectively) and low haematocrit (HTC) was associated with any hemoplasma infection $(P=0.004, \mathrm{OR}=0.34 ; 95 \% \mathrm{CI}: 0.13-0.91)$. These results are shown in Table 4.

Ten cats were seropositive to FIP $(3.29 \%, 10 / 304)$, with also one of these animals co-infected with CMhm and Mhf $(P=0.582$, OR $=1.25 ; 95 \%$ CI: $0.15-10.29)$, but 
Table 3 Distribution of feline hemoplasma infection in client-owned cats in accordance with different epidemiological data

\begin{tabular}{|c|c|c|c|c|c|c|c|c|}
\hline \multirow[t]{2}{*}{ Variable } & \multirow[t]{2}{*}{ Total no. of cats (\%) } & \multicolumn{7}{|c|}{ Number of positive cats (\%) } \\
\hline & & Any hemoplasma & Mhf & CMhm & $\mathrm{CMt}$ & Mhf + CMhm & $\mathrm{CMt}+\mathrm{CMhm}$ & $\mathrm{CMt}+\mathrm{Mhf}$ \\
\hline & 456 & $41(9.0)$ & $10(3.2)$ & $36(7.9)$ & $2(0.4)$ & $5(1.1)$ & $1(0.2)$ & $1(0.2)$ \\
\hline Age & 422 & & & & & & & \\
\hline Young ( $\leq 1$-year-old) & $85(20.1)$ & $2(2.3)$ & $2(2.3)$ & $2(2.3)$ & 0 & $2(2.3)$ & 0 & 0 \\
\hline Adult (> 1-year-old) & $337(79.9)$ & $31(9.2)^{*}$ & $6(1.8)$ & $27(8.0)$ & $1(0.3)$ & $2(0.6)$ & 0 & $1(0.3)$ \\
\hline Spayed/neutered & 397 & & & & & & & \\
\hline Yes & $247(62.2)$ & $22(8.9)$ & $4(1.6)$ & $20(8.1)$ & 0 & $2(0.8)$ & 0 & 0 \\
\hline No & $150(37.8)$ & $9(6.0)$ & $3(2.0)$ & $8(5.3)$ & $1(0.7)$ & $2(1.4)$ & 0 & $1(0.7)$ \\
\hline Breed & 424 & & & & & & & \\
\hline European & $294(69.3)$ & $26(8.8)$ & $6(2.0)$ & $22(7.5)$ & $1(0.3)$ & $2(0.7)$ & 0 & $1(0.3)$ \\
\hline Non-European & $130(30.7)$ & $9(6.9)$ & $3(2.3)$ & $8(6.1)$ & 0 & $2(1.5)$ & 0 & 0 \\
\hline Outdoor access & 333 & & & & & & & \\
\hline Yes & $82(24.6)$ & $10(12.2)^{*}$ & $1(1.2)$ & $9(11.0)$ & $1(1.2)$ & 0 & 0 & $1(1.2)$ \\
\hline No & $251(75.4)$ & $12(4.8)$ & $4(1.6)$ & $11(4.4)$ & 0 & $3(1.2)$ & 0 & 0 \\
\hline Contact with other animals & 331 & & & & & & & \\
\hline Yes & $225(68.0)$ & $18(8.0)$ & $2(0.9)$ & $17(7.6)$ & $1(0.4)$ & $1(0.4)$ & 0 & $1(0.4)$ \\
\hline No & $106(32.0)$ & $8(7.5)$ & $3(2.8)$ & $7(6.6)$ & 0 & $2(1.9)$ & 0 & 0 \\
\hline Previous tick infestation & 317 & & & & & & & \\
\hline Yes & $16(5.9)$ & 0 & 0 & 0 & 0 & 0 & 0 & 0 \\
\hline No & $301(94.1)$ & $21(7.0)$ & $5(1.7)$ & $19(6.3)$ & $1(0.3)$ & $3(1.0)$ & 0 & $1(0.3)$ \\
\hline Previous flea infestation & 316 & & & & & & & \\
\hline Yes & $41(13.0)$ & $4(9.8)$ & 0 & $4(9.8)$ & 0 & 0 & 0 & 0 \\
\hline No & $275(87.0)$ & $17(6.2)$ & $5(1.8)$ & $15(5.4)$ & $1(0.4)$ & $3(1.1)$ & 0 & $1(0.4)$ \\
\hline Ectoparasiticide treatment & 309 & & & & & & & \\
\hline Yes & $86(27.8)$ & $8(9.3)$ & $2(2.3)$ & $7(8.1)$ & 0 & $1(1.2)$ & 0 & 0 \\
\hline No & $223(72.2)$ & $13(5.8)$ & $3(1.3)$ & $12(5.4)$ & $1(0.4)$ & $2(0.9)$ & 0 & $1(0.4)$ \\
\hline Travel history & 309 & & & & & & & \\
\hline Yes & $116(37.5)$ & $7(6.0)$ & 0 & $7(6.0)$ & 0 & 0 & 0 & 0 \\
\hline No & $193(62.5)$ & $14(7.2)$ & $5(2.6)$ & $12(6.2)$ & $1(0.5)$ & $3(1.5)$ & 0 & $1(0.5)$ \\
\hline Previous blood transfusion & 314 & & & & & & & \\
\hline Yes & $4(1.3)$ & 0 & 0 & 0 & 0 & 0 & 0 & 0 \\
\hline No & $310(98.7)$ & $21(6.8)$ & $5(1.6)$ & $19(6.1)$ & $1(0.3)$ & $3(1.0)$ & 0 & $1(0.3)$ \\
\hline Tetracyclines treatment & 314 & & & & & & & \\
\hline Yes & $13(4.1)$ & $1(7.7)$ & 0 & $1(7.7)$ & 0 & 0 & 0 & 0 \\
\hline No & $301(95.9)$ & $20(6.6)$ & $5(1.7)$ & $18(6.0)$ & $1(0.3)$ & $3(1.0)$ & 0 & $1(0.3)$ \\
\hline
\end{tabular}

Abbreviations: CMhm "Candidatus Mycoplasma haemominutum", Mhf Mycoplasma haemofelis, CMt "Candidatus Mycoplasma turicensis"

*Statistically significant differences $(P<0.05)$

PCR for FIP was not performed. This animal was a young non-neutered male client-owned cat that was attended at the VMTH with musculoskeletal and gastrointestinal signs.

Data available for the two client-owned cats with CMt infection are also detailed in Tables 2, 3 and 4. One of them was co-infected with Mhf and the other with CMhm. The one co-infected with Mhf was a non- neutered adult male (3 years old), client-owned cat with outdoor access, living in a rural area and positive for FIV. It was found to have a slight monocytosis, without other abnormalities in blood analysis. No previous contact with ectoparasites was reported. Unfortunately, the data available for the client-owned cat co-infected with CMt and CMhm was very scarce. Finally, another coinfected cat with CMhm and CMt was a 15 year old 
Table 4 Distribution of feline hemoplasma infection in client-owned cats in accordance with different haematological findings, and the presence or absence of clinical signs

\begin{tabular}{|c|c|c|c|c|c|c|c|c|}
\hline \multirow[t]{2}{*}{ Variable } & \multirow{2}{*}{$\begin{array}{l}\text { Total no. } \\
\text { of cats } \\
(\%)\end{array}$} & \multicolumn{7}{|c|}{ Number of positive cats (\%) } \\
\hline & & Any hemoplasmas & Mhf & CMhm & $\mathrm{CMt}$ & $\mathrm{Mhf}+\mathrm{CMhm}$ & $\mathrm{CMt}+\mathrm{CMhm}$ & $\mathrm{CMt}+\mathrm{Mht}$ \\
\hline & 456 & $41(9.0)$ & $10(3.2)$ & $36(7.9)$ & $2(0.4)$ & $5(1.1)$ & $1(0.2)$ & $1(0.2)$ \\
\hline Clinical signs & 425 & & & & & & & \\
\hline Yes & $325(76.5)$ & $29(8.9)$ & $8(2.5)$ & $24(7.4)$ & $1(0.3)$ & $3(0.9)$ & 0 & $1(0.3)$ \\
\hline No & $100(23.5)$ & $5(5.0)$ & $1(1.0)$ & $5(5.0)$ & 0 & $1(1.0)$ & 0 & 0 \\
\hline Coronavirus seropositivity & 304 & & & & & & & \\
\hline Yes & $10(3.3)$ & $1(10.0)$ & $1(10.0)$ & $1(10.0)$ & 0 & $1(10.0)$ & 0 & 0 \\
\hline No & $294(96.7)$ & $24(8.2)$ & $6(2.0)$ & $21(7.1)$ & $1(0.3)$ & $3(1.0)$ & 0 & $1(0.3)$ \\
\hline \multicolumn{9}{|l|}{ Haematology } \\
\hline $\mathrm{RBC}\left(\times 10^{6} \mu \mathrm{l}\right)$ & 331 & & & & & & & \\
\hline High (> 10) & $60(18.1)$ & $4(6.7)$ & 0 & $4(6.7)$ & 0 & 0 & 0 & 0 \\
\hline Normal (5-10) & $251(75.8)$ & $23(9.2)$ & $3(1.2)$ & $21(8.4)$ & $2(0.8)$ & $1(0.4)$ & $1(0.4)$ & $1(0.4)$ \\
\hline $\operatorname{Low}(<5)$ & $20(6.0)$ & $6(30.0)^{*}$ & $2(10.0)^{*}$ & $5(25)^{*}$ & 0 & $1(5.0)$ & 0 & 0 \\
\hline $\mathrm{HGB}(\mathrm{g} / \mathrm{dl})$ & 407 & & & & & & & \\
\hline High (> 15) & $16(3.9)$ & $1(6.2)$ & 0 & $1(6.2)$ & 0 & $1(6.2)$ & 0 & 0 \\
\hline Normal (9-15) & 332 (81.6) & $26(7.8)$ & $6(1.8)$ & $23(6.9)$ & $2(0.6)$ & $3(0.9)$ & 0 & $1(0.3)$ \\
\hline $\operatorname{Low}(<9)$ & $59(14.5)$ & $12(20.3)^{*}$ & $2(3.4)$ & $11(18.6)^{*}$ & 0 & 0 & $1(1.7)$ & 0 \\
\hline Haematocrit (\%) & 410 & & & & & & & \\
\hline High (> 45) & $22(5.4)$ & $1(4.5)$ & 0 & $1(4.5)$ & 0 & 0 & 0 & 0 \\
\hline Normal (24-45) & $360(87.8)$ & $32(8.9)$ & $6(1.7)$ & $29(8.1)$ & $2(0.6)$ & $3(0.8)$ & $1(0.3)$ & $1(0.3)$ \\
\hline Low $(<24)$ & $28(6.8)$ & $6(21.4)^{*}$ & $2(7.1)$ & $5(17.9)$ & 0 & $1(3.6)$ & 0 & 0 \\
\hline MCV (fl) & 333 & & & & & & & \\
\hline High (> 55) & $5(1.5)$ & $2(40.0)$ & $1(20.0)$ & $1(20.0)$ & $1(20.0)$ & 0 & $1(20.0)$ & 0 \\
\hline Normal (39-55) & $252(75.7)$ & $25(9.9)$ & $4(1.6)$ & $23(9.1)$ & $1(0.4)$ & $2(0.8)$ & 0 & $1(0.4)$ \\
\hline Low $(<39)$ & 76 (22.8) & $4(7.9)$ & 0 & $6(7.9)$ & 0 & 0 & 0 & 0 \\
\hline $\mathrm{MCH}(\mathrm{pg})$ & 335 & & & & & & & \\
\hline High (> 17.5) & $7(2.1)$ & $2(28.6)$ & $1(12.5)$ & $1(14.3)$ & $1(12.5)$ & 0 & $1(12.5)$ & 0 \\
\hline Normal (12.5-17.5) & $273(81.5)$ & $27(9.9)$ & $3(1.1)$ & $25(9.2)$ & $1(0.4)$ & $1(0.4)$ & 0 & $1(0.4)$ \\
\hline Low $(<12.5)$ & $55(16.4)$ & $4(7.3)$ & $1(1.8)$ & $4(7.3)$ & 0 & $1(1.8)$ & 0 & 0 \\
\hline $\mathrm{MCHC}(\mathrm{g} / \mathrm{dl})$ & 405 & & & & & & & \\
\hline High (> 36) & $10(2.5)$ & $2(20.0)$ & $1(10.0)$ & $1(10.0)$ & $1(10.0)$ & 0 & 0 & $1(10.0)$ \\
\hline Normal (30-36) & $381(94.1)$ & $36(9.4)$ & $6(1.6)$ & $34(8.9)$ & $1(0.3)$ & $4(1.0)$ & $1(0.3)$ & 0 \\
\hline Low $(<30)$ & $14(3.5)$ & $1(7.1)$ & $1(7.1)$ & 0 & 0 & 0 & 0 & 0 \\
\hline Leukocytes $\left(\times 10^{3} \mu \mathrm{l}\right)$ & 408 & & & & & & & \\
\hline High (> 14) & 77 (18.9) & $5(6.5)$ & $1(1.3)$ & $5(6.5)$ & 0 & $1(1.3)$ & 0 & 0 \\
\hline Normal (5.5-14) & $273(66.9)$ & 29 (10.6) & $7(2.6)$ & $25(9.2)$ & $2(0.7)$ & $3(1.1)$ & $1(0.4)$ & $1(0.4)$ \\
\hline Low $(<5.5)$ & $58(14.2)$ & $5(8.6)$ & 0 & $5(8.6)$ & 0 & 0 & 0 & 0 \\
\hline Platelets $\left(\times 10^{3} \mu \mathrm{l}\right)$ & 148 & & & & & & & \\
\hline High $(>800)$ & $12(8.1)$ & 0 & 0 & 0 & 0 & 0 & 0 & 0 \\
\hline Normal (800-300) & $60(40.5)$ & $6(10.0)$ & $3(5.0)$ & $5(8.3)$ & 0 & $2(3.3)$ & 0 & 0 \\
\hline Low $(<300)$ & $76(51.3)$ & $7(9.2)$ & $1(1.3)$ & $7(9.2)$ & 0 & $1(1.3)$ & 0 & 0 \\
\hline
\end{tabular}


male stray cat, negative for retrovirus and with a high total protein concentration $(9.7 \mathrm{~g} / \mathrm{dl})$.

\section{Discussion}

The presence of hemotropic mycoplasma infections in cats from central Spain has been demonstrated in this study, with $\mathrm{CMhm}$ being the most prevalent species. The overall prevalence of hemoplasma infection in cats in the Madrid area determined in the current study $(10.6 \%$; 63/594) is comparable to the results of a previous study carried out in Barcelona, Spain [20], where $12 \%$ of a population of 191 cats with and without outdoor access was analysed. This hemotropic mycoplasma prevalence is also similar to those described in other studies performed in Denmark [23], Germany [24, 25], Italy [26] and Switzerland [10] and lower than the rates reported by others in Greece [27], Italy [28, 29] and Portugal $[6,30]$. These differences in prevalence can be due to several factors: the cat population sampled (which could present several risk factors simultaneously), geographical variations and/or differences in the diagnostic technique used in these studies (from microscopic to molecular detection among others). Our study was performed employing conventional PCR that has been highly employed previously and with good sensitivity, but it should be considered that a higher sensitivity could be achieved with a combination of real-time and conventional PCR analyses [5, 31, 32].

In agreement with our results, CMhm has been the most common hemoplasma found in the majority of prevalence studies carried out worldwide. This could be due, as suggested by Tanahara et al. [11], to a more efficient infection and multiplication of $\mathrm{CMhm}$ in comparison to other hemotropic mycoplasma, or to a lower virulence that allows a longer asymptomatic carrier state.

The presence of "Candidatus Mycoplasma haematoparvum" DNA has not been evaluated in this study. Considering the description of this infection in other countries [5-7], future work should be done to analyse this hemotropic mycoplasma species in central Spain.

Consistent with previous observations [6,33,34] of a higher prevalence of feline hemoplasma infection in countries with warmer climates, when analysing season of collection of blood samples in the current study, a statistically significant higher prevalence of hemoplasma infection during spring and summer was found, with most positive cats $(63.5 \%$ of positive samples, $40 / 63)$ included in the study between April and September. This seasonal influence on prevalence has been previously described when comparing summer with autumn in one study performed in Italy [28], suggesting vector transmission. However, it has not been proven for natural infection and other studies have not found any association between prevalence rate and season of the year [6, 28, 33, 34]. In fact, in the client-owned cat group of this study, history of previous ectoparasites infestation (ticks/fleas) was not found associated with hemoplasma infection, similarly to previous descriptions $[6,33]$. However, this information must be carefully assessed considering the natural behaviour of grooming in the cat, that could have facilitated the fact that arthropod infestation went unnoticed for the owner/person in charge of the cat [28]. In addition, other means of transmission must exist, considering their detection in areas where there is absence of the possible vectors [1]. In this sense, it has been described that male cats are more likely to engage in roaming and fighting behaviour, which may increase their chance of contracting the disease if a direct mode of transmission exists [7, 11, 20, 26, 33, 35-41]. Our own study supports a higher risk for male animals and for stray cats or client-owned cats that had outdoor access. All these factors may favour contact with other cats, although it should be considered that stray or client-owned cats with outdoor access are supposed to be more exposed not only to contact with other cats, but also to ectoparasites. In addition, it is important to consider the results of the logistic regression study carried out, that showed a stronger relationship of mycoplasma infection with the variable FIV status, which could mean that gender variable could be a confounding factor due to the relationship between positive FIV status and male sex.

The significant association between hemoplasma infection and the co-infection with retrovirus (FeLV/FIV) shown by this and previous studies $[6,19,25,35,39]$ could be explained by the well-known immunosuppressive effect of these retroviruses. However, since FIV is mainly transmitted through bite wounds, it has been suggested that the strong statistical association between this retroviral infection and feline hemoplasmosis could be supporting the horizontal route of transmission [20].

It has been frequently reported that there is an association between the age of the cats and hemoplasma infection. Some studies have described that adult age could be a risk factor for these infections $[7,11,23,26,33,35$, $40,41]$. In our study, being an adult cat (> one year-old) was statistically associated with hemoplasma infection. This association could be explained because older animals have been more exposed to blood-sucking arthropods and to more aggressive interactions with other cats. Moreover, this association between hemoplasma infection and adult cats could be explained by the existence of carrier cats with a chronic infection as described previously [42].

Clinical signs and laboratory findings detected for hemoplasma infection depend on a wide range of factors. Concurrent diseases or previous infections, hemoplasma species involved (CMhm, Mhf, CMt or even coinfections), and the stage (acuteness or chronicity) of hemoplasma infection could considerably change these 
findings. While the most frequently described clinical signs in cats with hemoplasmosis are related to the occurrence of anaemia, lethargy, or pale mucous membranes [1] among others, the current study showed statistical associations between the infection by CMhm and musculoskeletal and renal signs. These results should be interpreted with caution, considering that other diseases could be responsible for the clinical signs detected in the cats in the current study, as it was observed in the cat coinfected with FIP, CMhm and Mhf, that showed musculoskeletal and gastrointestinal signs. Sykes et al. [41] suggested a relation between renal signs and hemoplasma infection, but also considered that the infected population represented by old cats probably has a high prevalence of chronic kidney disease.

On the other hand, our study showed some remarkable laboratorial findings, such as low HTC, RBC count and HGB concentration. This and other studies [27] have detected hemoplasma infection both in cats suffering anaemia and in cats with red blood cells counts within reference values. Mhf is considered the most pathogenic feline hemoplasma species, causing extravascular erythrophagocytosis especially during the acute phase. However, an interesting finding to highlight is the association between the existence of anaemia not only in Mhf, but also in CMhm infected cats. It has been previously described that experimental CMhm infection generally does not induce anaemia and significant clinical signs, so geographical differences in the pathogenicity of the strain of this species should be considered. Nevertheless, it is important to note that the cats of the study could have been exposed to other pathogens previously described in central Spain $[43,44]$ or could be affected by other diseases associated with the development of anaemia.

This study also showed the presence of CMt in central Spain, although the low number of positive cats precludes a proper epidemiological analysis for this species.

\section{Conclusions}

To our knowledge, this is the first epidemiological survey of feline hemoplasmas performed in central Spain (Madrid). Our study confirms that the three-main species of hemoplasma are infecting client-owned and stray cats in this region of Spain, with " $\mathrm{Ca}$. Mycoplasma haemominutum" being the most prevalent species. Male gender, collection of blood during warm months, FeLV/ FIV positivity status, and outdoor access were associated with hemotropic mycoplasma infection in cats from Madrid. With regard to clinical signs and laboratory parameters, natural infection by feline hemoplasmas in the area is associated with anaemia, being difficult to determinate if co-infections can influence this finding. More studies are necessary to understand the role of the infection by these species of hemoplasma in cats.

\section{Abbreviations \\ Cl: Confidence interval; CMhm: "Candidatus Mycoplasma haemominutum"; CMt: "Candidatus Mycoplasma turicensis"; FCoV: Feline coronavirus; FeLV: Feline leukemia virus; FIP: Feline infectious peritonitis; FIV: Feline immunodeficiency virus; GAPDH: Gliceraldehide-3-phosphate dehydrogenase HGB: Haemoglobin concentration; Mhf: Mycoplasma haemofelis; MCH: Mean corpuscular haemoglobin; MCHC: Mean corpuscular haemoglobin concentration; MCV: Mean corpuscular haemoglobin; OR: Odds ratio; PCR: Polymerase chain reaction; RBC: Red blood cell count; RNA: Ribonucleic acid; VMTH: Veterinary Medicine Teaching Hospital}

\section{Acknowledgements}

Publication of this paper has been sponsored by Bayer Animal Health in the framework of the 13th CVBD World Forum Symposium.

\section{Funding}

DDR was supported by the European Social Fund (PAl-grant PEJ15/ BIO/AI-0327).

\section{Availability of data and materials}

All data generated or analysed during this study are included in this published article.

\section{Authors' contributions}

DDR performed laboratory work, analysed data and wrote the manuscript. AV designed and supervised the study, participated in data analysis, and wrote the manuscript. TA, FRF, MCG and BA collected samples, participated in data analysis and in writing the manuscript. AS planned and supervised the study, analysed data and assisted in writing the manuscript. All authors read and approved the final manuscript.

Ethics approval and consent to participate

Written consent for patient inclusion in this study was obtained for every case. In addition, protocols approved by the Animal Experimentation

Committee of the institution were always followed.

Consent for publication

Not applicable.

Competing interests

The authors declare that they have no competing interests.

\section{Publisher's Note}

Springer Nature remains neutral with regard to jurisdictional claims in published maps and institutional affiliations.

\section{Author details}

${ }^{1}$ Department of Animal Medicine and Surgery, College of Veterinary Medicine, Complutense University of Madrid, Avda. Puerta de Hierro s/n, 28040 Madrid, Spain. ${ }^{2}$ Instituto Nacional de Infectología Evandro Chagas, Fiocruz, Avenida Brazil 4365, CEP 21040- 900, Rio de Janeiro, Brazil.

Received: 15 January 2018 Accepted: 22 February 2018

Published online: 20 March 2018

\section{References}

1. Tasker S. Haemotropic mycoplasmas: what's their real significance in cats? J Feline Med Surg. 2010;(5):369-81.

2. Neimark H, Johansson K-E, Rikihisa Y, Tully JG. Proposal to transfer some members of the genera Haemobartonella and Eperythrozoon to the genus Mycoplasma with descriptions of "Candidatus Mycoplasma haemofelis" "Candidatus Mycoplasma haemomuris", "Candidatus Mycoplasma haemosuis" and "Candidatus Mycoplasma wenyonii". Int J Syst Evol Microbiol. 2001:51:891-9.

3. Foley JE, Pedersen NC. "Candidatus Mycoplasma haemominutum", a lowvirulence epierythrocytic parasite of cats. Int J Syst Evol Microbiol. 2001;51: 815-7. 
4. Willi B, Boretti FS, Cattori V, Tasker S, Meli ML, Reusch C, et al. Identification, molecular characterization, and experimental transmission of a new hemoplasma isolate from a cat with hemolytic anemia in Switzerland. J Clin Microbiol. 2005:43:2581-5.

5. Sykes JE, Drazenovich NL, Ball LM, Leutenegger CM. Use of conventional and real-time polymerase chain reaction to determine the epidemiology of hemoplasma infections in anemic and nonanemic cats. J Vet Intern Med. 2007;21:685-93.

6. Martínez-Díaz VL, Silvestre-Ferreira AC, Vilhena H, Pastor J, Francino O, Altet L. Prevalence and co-infection of haemotropic mycoplasmas in Portuguese cat by real-time polymerase chain reaction. J Feline Med Surg. 2013;15:879-85.

7. Vergara RW, Galleguillos FM, Jaramillo MG, Almosny NRP, Martínez PA, Behne PG, et al. Prevalence, risk factor analysis, and hematological findings of hemoplasma infection in domestic cats from Valdivia, southern Chile. Comp Immunol Microbiol Infect Dis. 2016;46:20-6.

8. Jensen WA, Lappin MR, Kamkar S, Reagan WJ. Use of a polymerase chain reaction assay to detect and differentiate two strains of Haemobartonella felis in naturally infected cats. Am J Vet Res. 2001;(4):604-8.

9. Criado-Fornelio A, Martinez-Marcos A, Buling-Sarana A, Barba-Carretero J. Presence of Mycoplasma haemofelis, Mycoplasma haemominutum and piroplasmids in cats from southern Europe: a molecular study. Vet Microbiol. 2003;93:307-17.

10. Willi B, Boretti FS, Baumgartner C, Tasker S, Wenger B, Cattori V, et al. Prevalence, risk factor analysis, and follow-up of infections caused by three feline hemoplasma species in cats in Switzerland. J Clin Microbiol. 2006:44:961-9.

11. Tanahara M, Miyamoto S, Nishio T, Yoshii Y, Sakuma M, Sakata Y, et al. An epidemiological survey of feline hemoplasma infection in Japan. J Vet Med Sci. 2010;72:1575-81.

12. Kamrani A, Parreira VR, Greenwood J, Prescott JF. The prevalence of Bartonella, hemoplasma, and Rickettsia felis infections in domestic cats and in cat fleas in Ontario. Can J Vet Res. 2008;72:411-9.

13. Shaw S, Kenny M, Tasker S, Birtles R. Pathogen carriage by the cat flea Ctenocephalides felis (Bouché) in the United Kingdom. Vet Microbiol. 2004; 102:183-8.

14. Lappin MR, Griffin B, Brunt J, Riley A, Burney D, Hawley J, et al. Prevalence of Bartonella species, haemoplasma species, Ehrlichia species, Anaplasma phagocytophilum, and Neorickettsia risticii DNA in the blood of cats and their fleas in the United States. J Feline Med Surg. 2006;8:85-90.

15. Hornok S, Meli ML, Perreten A, Farkas R, Willi B, Beugnet F, et al. Molecular investigation of hard ticks (Acari: Ixodidae) and fleas (Siphonaptera: Pulicidae) as potential vectors of rickettsial and mycoplasmal agents. Vet Microbiol. 2010;140:98-104.

16. Mendes-de-Almeida F, Labarthe N, Guerrero J, Faria MCF, Branco AS, Pereira $C D$, et al. Follow-up of the health conditions of an urban colony of freeroaming cats (Felis catus Linnaeus, 1758) in the city of Rio de Janeiro, Brazil. Vet Parasitol. 2007:147:9-15.

17. Willi B, Boretti FS, Meli ML, Bernasconi MV, Casati S, Hegglin D, et al. Realtime PCR investigation of potential vectors, reservoirs, and shedding patterns of feline hemotropic mycoplasmas. Appl Environ Microbiol. 2007; 73:3798-802.

18. Taroura S, Shimada Y, Sakata Y, Miyama T, Hiraoka H, Watanabe M, et al. Detection of DNA of "Candidatus Mycoplasma haemominutum" and Spiroplasma sp. in unfed ticks collected from vegetation in Japan. J Vet Med Sci. 2005;67:1277-9.

19. Sykes JE, Terry JC, Lindsay LL, Owens SD. Prevalences of various hemoplasma species among cats in the United States with possible hemoplasmosis. J Am Vet Med Assoc. 2008:232:372-9.

20. Roura X, Peters IR, Altet $L$, Tabar M-D, Barker EN, Planellas $M$, et al. Prevalence of hemotropic mycoplasmas in healthy and unhealthy cats and dogs in Spain. J Vet Diagn Invest. 2010;22:270-4.

21. Ravicini S, Pastor J, Hawley J, Brewer M, Castro-López J, Beall M, et al. Prevalence of selected infectious disease agents in stray cats in Catalonia, Spain. JFMS Open Rep. 2016;2:1-6. 2055116916634109

22. Birkenheuer AJ, Levy MG, Breitschwerdt EB. Development and evaluation of a seminested PCR for detection and differentiation of Babesia gibsoni (Asian genotype) and B. canis DNA in canine blood samples. J Clin Microbiol. 2003; 41:4172-7.

23. Rosenqvist MB, Meilstrup A-KH, Larsen J, Olsen JE, Jensen AL, Thomsen LE. Prevalence of feline haemoplasma in cats in Denmark. Acta Vet Scand. 2016; $58: 78$.
24. Just F, Pfister K. Detection frequency of haemoplasma infections of the domestic cat in Germany. Berl Munch Tierarztl Wochenschr. 2007;120: 197-201.

25. Bauer N, Balzer H-J, Thüre S, Moritz A. Prevalence of feline haemotropic mycoplasmas in convenience samples of cats in Germany. J Feline Med Surg. 2008;10:252-8.

26. Ravagnan S, Carli E, Piseddu E, Da Rold G, Porcellato E, Zanardello C, et al. Prevalence and molecular characterization of canine and feline hemotropic mycoplasmas (hemoplasmas) in northern Italy. Parasit Vectors. 2017;10:132.

27. Maher IE, Tasker S, Polizopoulou Z, Dasopoulou A, Egan K, Helps CR, et al. Polymerase chain reaction survey of feline haemoplasma infections in Greece. J Feline Med Surg. 2010;12:601-5.

28. Gentilini F, Novacco M, Turba ME, Willi B, Bacci ML, Hofmann-Lehmann R. Use of combined conventional and real-time PCR to determine the epidemiology of feline haemoplasma infections in northern Italy. J Feline Med Surg. 2009;11:277-85.

29. Spada E, Proverbio D, Galluzzo P, Della Pepa A, Bagnagatti De Giorgi G, Perego $R$, et al. Prevalence of haemoplasma infections in stray cats in northern Italy. ISRN Microbiol. 2014(2014);298352.

30. Duarte A, Marques V, Correia JHD, Neto I, Bráz BS, Rodrigues C, et al. Molecular detection of haemotropic Mycoplasma species in urban and rural cats from Portugal. J Feline Med Surg. 2015:17:516-22.

31. Watanabe M, Hisaue M, Hashizaki K, Furuichi M, Ogata M, Hisamtsu S, et al. Molecular detection and characterization of Haemobartonella felis in domestic cats in Japan employing sequence-specific polymerase chain reaction (SS-PCR). J Vet Med Sci. 2003:65:1111-4

32. Peters IR, Helps CR, Willi B, Hofmann-Lehmann R, Tasker $S$. The prevalence of three species of feline haemoplasmas in samples submitted to a diagnostics service as determined by three novel real-time duplex PCR assays. Vet Microbiol. 2008;126:142-50.

33. Silaghi C, Knaus M, Rapti D, Kusi I, Shukullari E, Hamel D, et al. Survey of Toxoplasma gondii and Neospora caninum, haemotropic mycoplasmas and other arthropod-borne pathogens in cats from Albania. Parasit Vectors. $2014 ; 7: 62$

34. Laberke S, Just F, Pfister K, Hartmann K. Prevalence of feline haemoplasma infection in cats in Southern Bavaria, Germany, and infection risk factor analysis. Berl Munch Tierarztl Wochenschr. 2010;123:42-8.

35. Bergmann M, Englert T, Stuetzer B, Hawley JR, Lappin MR, Hartmann K. Risk factors of different hemoplasma species infections in cats. BMC Vet Res. 2017;13:52.

36. Jenkins KS, Dittmer KE, Marshall JC, Tasker S. Prevalence and risk factor analysis of feline haemoplasma infection in New Zealand domestic cats using a real-time PCR assay. J Feline Med Surg. 2013;15:1063-9.

37. Hwang J, Gottdenker N, Min M-S, Lee H, Chun M-S. Evaluation of biochemical and haematological parameters and prevalence of selected pathogens in feral cats from urban and rural habitats in South Korea. J Feline Med Surg. 2016;18:443-51.

38. Aquino LC, Hicks CA, Scalon MC, Lima MGM, Lemos MS, Paludo GR, et al, Prevalence and phylogenetic analysis of haemoplasmas from cats infected with multiple species. J Microbiol Methods. 2014;107:189-96.

39. Stojanovic V, Foley P. Infectious disease prevalence in a feral cat population on Prince Edward Island, Canada. Can Vet \. 2011;52:979-82.

40. Assarasakorn S, Veir J, Hawley J, Brewer M, Morris A, Hill A, et al. Prevalence of Bartonella species, hemoplasmas, and Rickettsia felis DNA in blood and fleas of cats in Bangkok, Thailand. Res Vet Sci. 2012;93:1213-6.

41. Sykes JE. Feline hemotropic mycoplasmas. J Vet Emerg Crit Care (San Antonio). 2010;20:62-9.

42. Santos AP, Conrado FO, Messick JB, Biondo AW, STd O, Guimaraes AMS, et al. Hemoplasma prevalence and hematological abnormalities associated with infection in three different cat populations from southern Brazil. Rev Bras Parasitol Vet. 2014:23:428-34.

43. Ayllón T, Diniz PP, Breitschwerdt E, Villaescusa A, Rodríguez-Franco F, Sainz A. Vector-borne diseases in client-owned and stray cats from Madrid, Spain. Vector Borne Zoonotic Dis. 2012:12:143-50.

44. Díaz-Regañón D, Villaescusa A, Ayllón T, Rodríguez-Franco F, Baneth G, Calleja-Bueno L, et al. Molecular detection of Hepatozoon spp. and Cytauxzoon sp. in domestic and stray cats from Madrid, Spain. Parasit vectors. 2017:10:112. 